\title{
Destruction of DNA-binding proteins by programmable O'PROTAC: Oligonucleotide-based PROTAC
}

Jingwei Shao ${ }^{1,5}$, Yuqian Yan ${ }^{2,5}$, Donglin Ding ${ }^{2,5}$, Dejie Wang ${ }^{2}$, Yundong $\mathrm{He}^{2}$, Yunqian

Pan $^{2}$, Wei Yan ${ }^{1}$, Anupreet Kharbanda ${ }^{1}$, Hong-yu Li ${ }^{1 *}$ and Haojie Huang ${ }^{2,3,4 *}$

${ }^{1}$ Department of Pharmaceutical Sciences, College of Pharmacy, University of

Arkansas for Medical Sciences, Little Rock, AR 72205, USA

${ }^{2}$ Department of Biochemistry and Molecular Biology, Mayo Clinic College of

Medicine and Science, Rochester, MN 55905, USA

${ }^{3}$ Department of Urology, Mayo Clinic College of Medicine and Science, Rochester, MN 55905, USA

${ }^{4}$ Mayo Clinic Cancer Center, Mayo Clinic College of Medicine and Science, Rochester, MN 55905, USA.

${ }^{5}$ These authors contributed equally.

\section{* Corresponding authors}

Hong-yu Li (HLi2@uams.edu) and Haojie Huang (huang.haojie@mayo.edu) 


\begin{abstract}
DNA-binding proteins including transcription factors (TFs) play essential roles in gene transcription and DNA replication and repair during normal organ development and pathogenesis of diseases such as cancer, cardiovascular disease and obesity, deeming to be a large repertoire of attractive therapeutic targets. However, this group of proteins are generally considered undruggable as they lack an enzymatic catalytic site or a ligand binding pocket. PROteolysis-TArgeting Chimera (PROTAC) technology has been developed by engineering a bifunctional small molecule chimera to bring a protein of interest (POI) to the proximity of an E3 ubiquitin ligase, thus inducing the ubiquitination of POI and further degradation through proteasome pathway. Here we report the development of Oligonucleotide-based PROTAC (O'PROTACs), a class of noncanonical PROTACs in which a TF-recognizing doublestranded oligonucleotide is incorporated as a binding moiety of POI. We demonstrate that O'PROTACs of ERG and LEF1, two highly cancer-related transcription factors selectively promote degradation of these proteins and inhibit their transcriptional activity in cancer cells. The programmable nature of O'PROTACs indicates that this approach is applicable to destruct other TFs. O'PROTACs not only can serve as a research tool, but also can be harnessed as a therapeutic arsenal to target DNA binding proteins for effective treatment of diseases such as cancer.
\end{abstract}

\title{
Introduction
}

A large group of DNA-binding proteins act as transcription factors (TFs) that 
transcriptionally activate or suppress gene expression by interacting with specific

DNA sequence and transcription co-regulators. Approximately 2,000 TFs have been

identified in eukaryotic cells and they are associated with numerous biological

processes. Among them, approximately 294 TFs are associated with cancer

development, which account for $\sim 19 \%$ of oncogenes ${ }^{1}$. Therefore, targeting TFs

associated with cancer development appears to be an appealing strategy for cancer

treatment.

In the last decades, small molecule modulators have been developed to target nuclear receptors on the basis that this class of TFs contains a clearly defined ligand-

binding pocket ${ }^{2}$. However, most of other TFs are difficult to target because they lack a ligand binding pocket. As the knowledge regarding the mechanisms of the assembly of transcription complexes has increased exponentially, different strategies to modulate the activity of TFs with small molecule compounds have emerged, including blocking protein/protein interactions, protein/DNA interactions, or chromatin remodeling/epigenetic reader proteins ${ }^{3}$. However, the development of traditional small molecules inhibiting non-ligand TFs remains very challenging, and a new targeting strategy to overcome the hurdle is highly demanded.

PROTACs are heterobifunctional small molecules composed of a POI ligand as a warhead, a linker and an E3 ligase ligand. The PROTAC molecule recruits the E3 ligase to the POI and induced the ubiquitination of the latter and further degradation by the proteasome pathway. PROTAC technology has greatly advanced during the last decade. It has been proved that PROTACs are capable of degrading a variety of 
proteins, including enzymes and receptors ${ }^{4-7}$. Two PROTACs, ARV-110 and ARV-471 which are androgen receptor (AR) and estrogen receptor (ER) degraders, respectively have entered phase I clinical trials ${ }^{8-9}$. PROTACs offer several advantages over small molecule inhibitors including expanding target scope, improving selectivity, reducing toxicity and evading inhibitor resistance ${ }^{10}$. This suggests that PROTAC technology is a new promising modality to tackle diseases, in particular for cancer. Most recently, PROTACs have been designed to degrade TFs. Wang's group developed a potent and signal transducers and activators of transcription 3 (STAT3)-specific degrader based on an STAT3 inhibitor SI-109 and demonstrated its targeting efficacy in vivo ${ }^{11}$. Crews' group reported the development of Transcription Factor Targeting Chimeras (TRAFTACs) $)^{12}$, which utilize haloPROTAC, dCas9-HT7 and dsDNA/CRISPR-RNA chimeras to degrade TFs. Nevertheless, this approach uses the artificially engineered dCas9-HT7 fusion protein as a mediator, which limits its potential use in clinic.

ERG transcription factor belongs to the ETS family and is involved in bone development, hematopoiesis, angiogenesis, vasculogenesis, inflammation, migration and invasion ${ }^{13}$. Importantly, it is overexpressed in approximately $50 \%$ of all human prostate cancer cases including both primary and metastatic prostate cancer due to the fusion of $E R G$ gene with the androgen-responsive TMPRSS2 gene promoter ${ }^{14}$. TMPRSS2-ERG gene fusion results in aberrant overexpression of truncated ERG, implying that increased expression of ERG is a key factor to drive prostate cancer progression $^{15}$. Therefore, therapeutic targeting ERG is urgently needed to effectively treat prostate cancer patients. Lymphoid enhancer-binding factor 1 (LEF1) is another 
highly cancer-related TF. It belongs to T cell factor (TCF)/ LEF1 family. Complexed with $\beta$-catenin, LEF1 promotes the transcription of Wnt target genes ${ }^{16}$. LEF1 also can facilitate epithelial-mesenchymal transition $(\mathrm{EMT})^{17}$. Aberrant expression of LEF1 is implicated in several cancer types and related to cancer cell proliferation, migration, and invasion ${ }^{18}$. Hence, LEF1 is another ideal target for cancer treatment.

In the present study, we introduce a new strategy to target TFs using O'PROTACs, in which a double-stranded oligonucleotide is incorporated as POI binding moiety in PROTAC (Figure 1). We demonstrate that ERG O'PROTAC promotes proteasomal degradation of ERG protein and inhibits ERG transcriptional activity. Akin to ERG degrader, LEF1 O'PROTAC induces the degradation of LEF1. Consequently, its target gene expression and prostate cancer cell growth was also effectively inhibited.

\section{Results}

\section{Design of O'PROTACs}

ERG recognizes a highly conserved DNA binding consensus sequence including the 5'-GGAA/T-3' core motif ${ }^{19}$. We designed a 19-mer double-stranded oligonucleotide containing the sequence of ACGGACCGGAAATCCGGTT with the ERG binding moiety underscored. As for the E3 ligase-recruiting element, we selected the widely used pomalidomide and VHL-032, which are capable of hijacking cereblon and von Hippel-Lindau (VHL) respectively. PROTAC exerts its function based on the formation of ternary complex, in which a linker plays an important role. 
Therefore, we designed and synthesized six phosphoramidites with different linkers in different lengths and types, three of which are linked to pomalidomide and three with VHL-032 (P1-6, Table 1). The phosphoramidite was attached to the 5' terminal of one DNA strand through DNA synthesizer. After annealing, we generated six O’PROTACs (OPs) for both ERG and LEF1. (Table S1).

\section{Chemical synthesis of P1-6}

The synthesis of P1-6 was illustrated in Scheme 1. 4-Fluoro-thalidomide and VHL032 were prepared according to literature procedures ${ }^{20-21}$. The straightforward nucleophilic aromatic substitution reaction of 4-fluoro-thalidomide with different amines provided key intermediates 8a-c. VHL-032 was coupled with various carboxylic acids containing TBDPS protected hydroxyl group to deliver intermediates 8d-f. Subsequent acetylation of the hydroxyl groups in 8d-f and removal of the TBDPS protection produced intermediates 10a-c. Phosphitylation of 8a-c or 10a-c with Cl-POCEN ${ }^{i} \operatorname{Pr}_{2}$ yielded P1-6 in the presence of DIPEA.

\section{ERG O'PROTACs promote proteasome degradation of WT and TMPRSS2-}

\section{ERG proteins}

The nucleic acid-based agents typically rely on lipid-mediated transfection to deliver them into cells. FITC-labelled ERG O'PROTAC was synthesized to determine the transfection efficiency under a fluorescent microscope. We transfected 293T cells with 100 or $1,000 \mathrm{nM}$ of O'PROTAC with or without lipofectamine 2000. As 
expected, the presence of lipofectamine greatly enhanced the cellular uptake comparing with mock transfection (Figure 2A). However, there was no difference in uptake efficacy between low (100 nM) and high concentration (1,000 nM) (Figure 2A), probably owing to the saturation of the positively charged lipid with negatively charged oligonucleotide.

To assess the effects of ERG O'PROTACs on ERG proteins in cells, 293T cells were transfected with exogenously expressing HA-ERG plasmid and six ERG O'PROTACs at $100 \mathrm{nM}$ for 48 hours and ERG protein level was measured by western blot. A significant decrease in ERG protein level was observed upon treatment with ERG OP-C1-3 attached with pomalidomide while the effects of ERG OP-V1-3 conjugated with VHL-032 were much modest (Figure 2B). To further demonstrate the cellular effect on endogenous ERG protein level, we tested ERG O'PROTACs in ERG-overexpressed human prostate cancer cell line VCaP which expresses both fulllength ERG and TMPRSS2-ERG truncation ${ }^{22}$. Similar to the effect on ectopically expressed ERG, ERG OP-C1-3 also effectively decreased endogenous ERG protein in VCaP cells (Figure 2C). These data also imply that a shorter linker such as five carbon atoms (ERG OP-C1) might favorably form a more stable ternary complex. Although ERG OP-C1 significantly decreased ERG protein level, proteinase inhibitor MG132 blocked this degradation (Figure 3), suggesting ERG O'PROTAC degrades ERG protein via proteasome pathway.

In vitro biotin pulldown assay showed that a significant amount of HA-ERG expressed in $293 \mathrm{~T}$ cells was pulled down by biotin-labelled ERG OP-C1 and OP-C2 
(Figure 4), indicating that these two O'PROTACs strongly interact with ERG protein.

This result also provides a plausible explanation for the better effect of these two O'PROTACS on ERG degradation.

Time-course studies showed that ERG O'PROTACs took effects starting from 12 hours until 48 hours examined (Figure 5A). Consistent with the finding in $293 \mathrm{~T}$ cells (Figure 2A), the dose-course experiments revealed that $100 \mathrm{nM}$ of ERG OP-C1 showed a significant inhibition of ERG protein level and this effect was not improved by higher concentrations such as 500 and $1,000 \mathrm{nM}$, indicating that ERG OP-C1 is probably saturated in a higher concentration (Figure 5B). Additionally, treatment of VCaP cells with ERG OP-C1 inhibited mRNA expression of ERG target genes including $A D A M 19, M M P 3, M M P 9, P L A T$ and $P L A U$ (Figure 5C), suggesting that ERG O'PROTAC inhibits ERG transcriptional activity in VCaP prostate cancer cells.

\section{Targeting other TFs for degradation by O'PROTACs}

To extend the utility of O'PROTACs, we turned to another transcription factor

LEF1. LEF1 acts as a DNA binding subunit in the $\beta$-catenin/LEF1 complex and exerts transcriptional regulation via binding to the nucleotide sequence 5'-A/TA/TCAAAG$3^{2}{ }^{23}$. We designed 18-mer double-stranded oligonucleotide containing the sequence of TACAAAGATCAAAGGGTT as the LEF1 binding moiety. Six LEF1 O’PROTACs

(Table S1) were synthesized using the same protocol as for the ERG O'PROTACs.

We first evaluated the degradation capability of each LEF1 O'PROTACs in PC3 prostate cancer cell line. Western blot assay was utilized to detect the expression of 
LEF1 protein. As shown in Figure 6, LEF1 OP-V1 potently induced LEF1 degradation in PC-3 cells at a lower concentration (100 nM) while other LEF1 O'PROTACs were less or not active. This result is similar with ERG O'PROTACs, suggesting that both linker length and E3 ligase are important factors for degradation of a specific TF.

Next, we examined the effect of LEF1 O'PROTAC on the transcriptional activity of the $\beta$-Catenin/LEF1 complex. We found that treatment of PC-3 prostate cancer cells with LEF1 OP-V1 downregulated mRNA expression of CCND1 and $c$ $M Y C$, two target genes of $\beta$-Catenin/LEF1 in a dose-dependent manner (Figure 7A and B). While LEF1 OP-V1 treatment did not affect mRNA expression of $L E F 1$ gene, it markedly decreased expression of LEF1 and its target protein Cyclin D1 at the protein level in PC-3 (Figure 7A). Importantly, LEF1 OP-V1 significantly inhibited PC-3 cell growth in a time- and dose-dependent fashion (Figure 7A and C). Similar results were obtained in another prostate cancer cell line DU145 (Figure 7D-F).

Collectively, LEF1 OP-V1 is a potent LEF1 degrader.

\section{Discussion}

In this study we take a new strategy of degrading "undruggable" transcription factors by employing O'PROTACs. O'PROTAC exploits natural "ligand" of transcription factors, namely specific DNA sequence, attached to an E3 ligase ligand via a linker. The tactic has been successfully applied to degrade ERG and LEF1 TFs with potent efficacy in cultured cells. 
Conventional PROTAC technology is rapidly evolving with some of them are in clinical trials; however, it inherits certain limitations. First, most of the reported PROTACs rely on the existing small molecules as POI targeting warhead, which make it difficult to be applied to "undruggable" targets like TFs. Additionally, due to their high molecular weight (600 1400 Da), PROTACs suffer from poor cell permeability, stability and solubility ${ }^{24}$. In comparison with classic small molecule drugs, PROTACs are significantly less druggable. O'PROTACs hold enormous potentials to transcend the limitations of conventional PROTACs. Because of their modalities, degraders can be rationally programmed according to the DNA binding sequence of a given TF, thus theoretically making it possible to target any TF of interest. Our data suggest that the efficacy of O'PROTACs can be further optimized by altering the lengths and types of a linker and the E3 ligase ligand. Moreover, the synthesis of O'PROTAC is highly simple and efficient, which facilitates the rapid development of a O'PROTAC library for high-throughput screening of the most potent TF degraders. O'PROTAC could be applied to any proteins bound to DNA duplexes.

Hall and colleagues recently report RNA-PROTACs, which utilize singlestranded RNA (ssRNA) to recruit RNA-binding protein (RBP). The binding of RBP with RNA heavily relies on both sequence motif and secondary structure ${ }^{25-26}$. Predicting the interaction between RNA and RBP is challenging due to the high flexibility of RNA ${ }^{27-28}$. However, double-stranded DNA bear a well-defined threedimensional duplex structure; therefore, the protein binding region is accessible and 
predictable. Hence, O'PROTAC is programmable by changing the nucleotide sequence that binds protein. Additionally, compared with double-stranded oligonucleotide, ssRNA is susceptible to deleterious chemical or enzymatic attacks ${ }^{28}$. Taken together, O'PROTAC is desirable due to its readily predictability and superior stability.

Oligonucleotide drug development has become a main stream for new drug hunting in the last decade ${ }^{29}$. The catalytic advantage of PROTACs ${ }^{30}$ incorporated into oligonucleotide drugs could further fuel the field. Moreover, the delivery of oligonucleotide drugs has been advanced significantly in the recent years, notably for mRNA COVID-19 vaccine ${ }^{31-32}$. Therefore, O'PROTACs can be a complementary drug discovery and development platform to conventional PROTACs to derive clinical candidates and accelerate drug discovery.

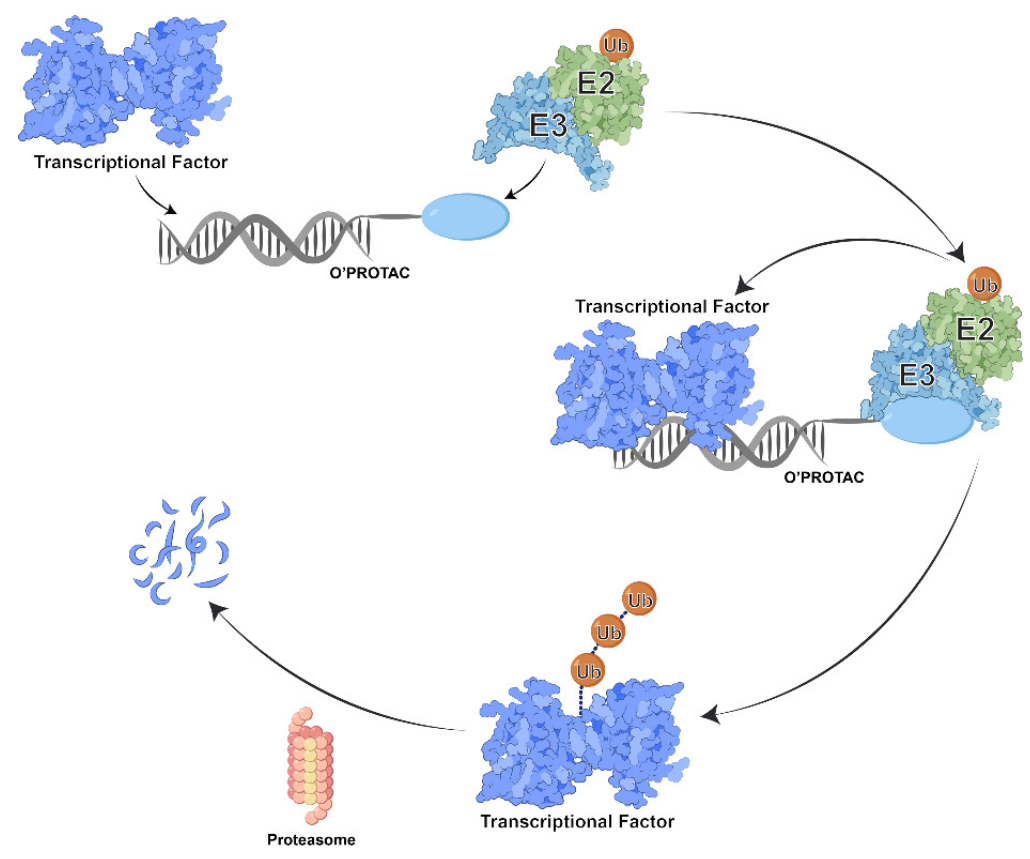

Fig 1. Schematic depicting the working principle of O'PROTAC. 
Table 1. The structures of phosphoramidites P1-6.

Cmpd Structure

\section{Scheme 1. Synthesis of P1-6}

A

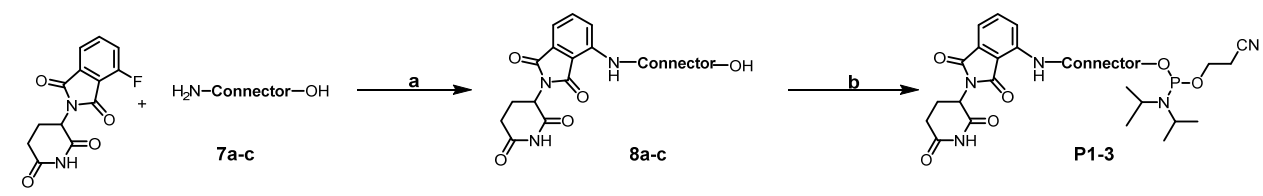

B

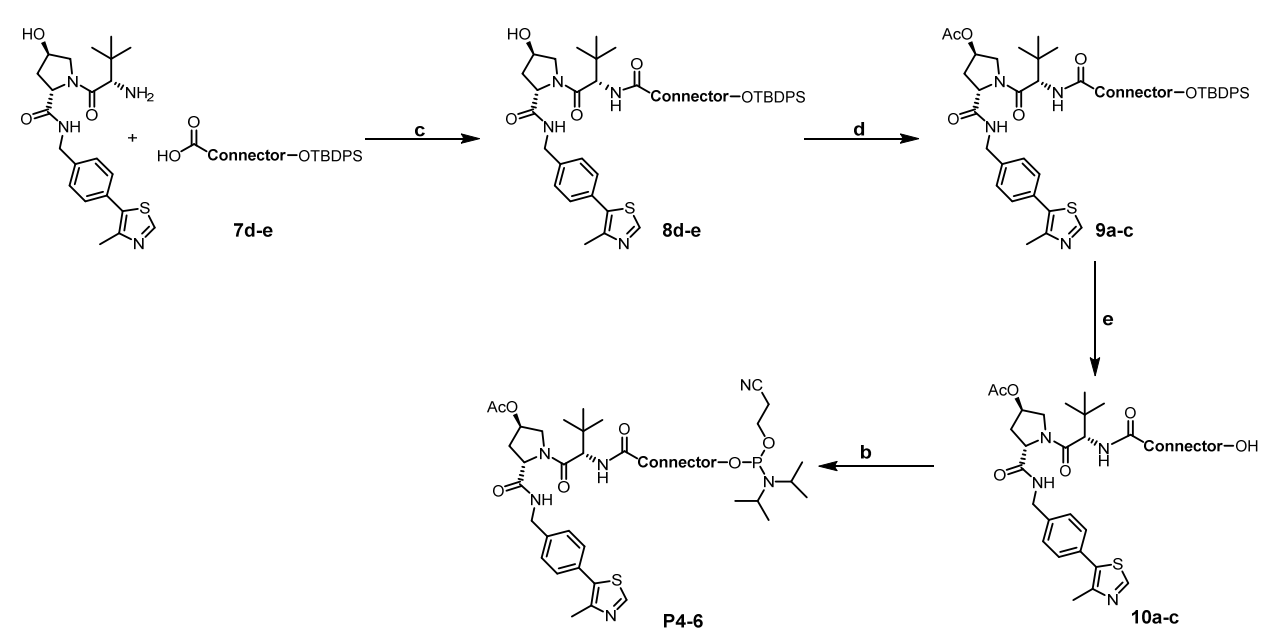

${ }^{a}$ Reagents and conditions: (a) DIPEA, NMP, MW, $100^{\circ} \mathrm{C}, 3 \mathrm{~h}$; (b) Cl-POCEN ${ }^{i} \mathrm{Pr}_{2}$, DIPEA, 
DCM, 1 h, rt. (c) HATU, TEA, DMF; (d) Ac $2 \mathrm{O}$, DMAP, DCM, 1 h; (e) TBAF, THF.

A

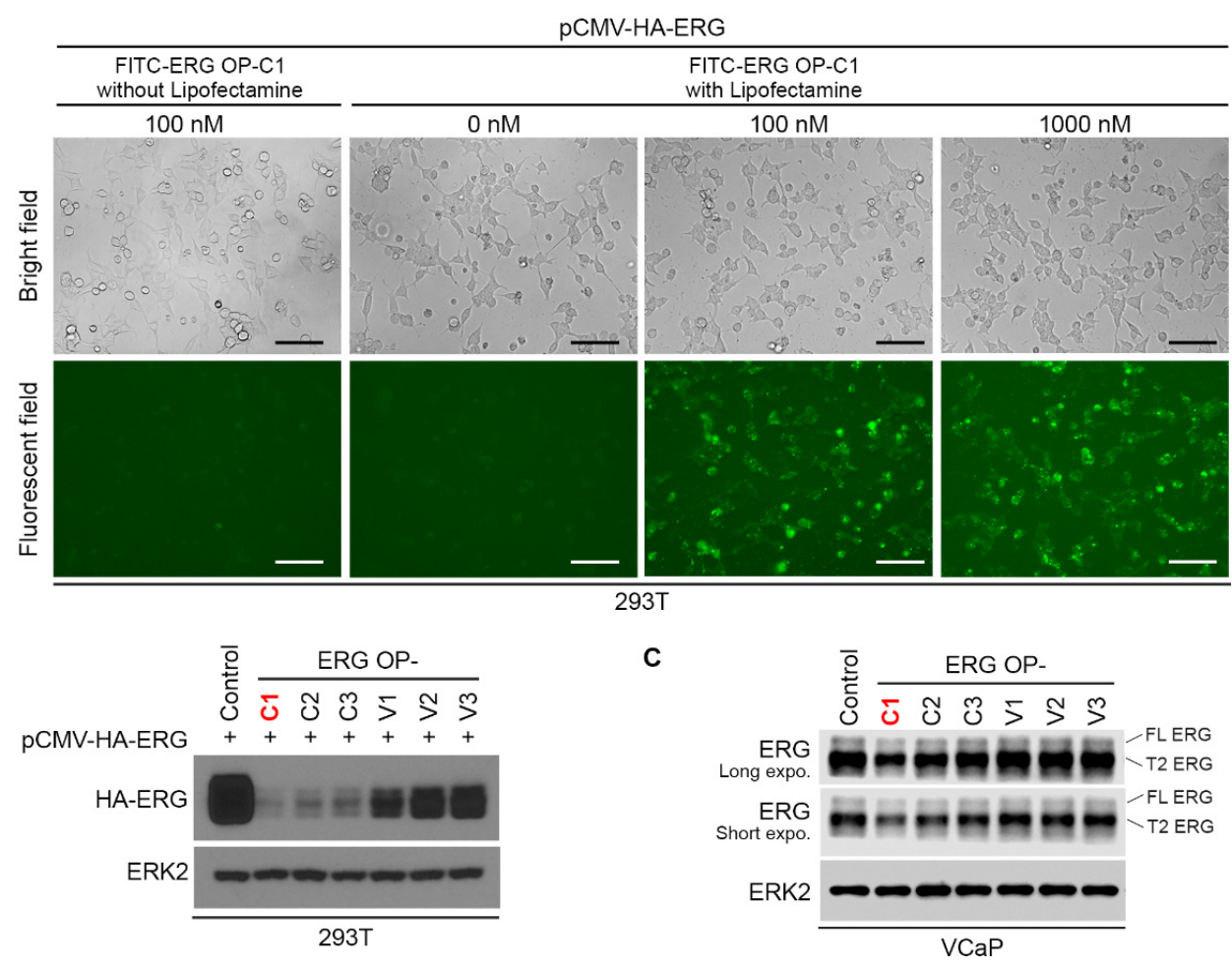

Figure 2. ERG O'PROTACs degrade ERG protein in cultured cells. (A) 293T

cells were transfected with FITC-labelled ERG O'PROTAC-13 (100 nM and 1,000

$\mathrm{nM}$ ) and the transfection efficiency was monitored $48 \mathrm{~h}$ post-transfection using a

fluorescent microscope. Scale bar: $50 \mu \mathrm{m}$. (B) $293 \mathrm{~T}$ cells were transfected with HA-

ERG plasmid and a control or six indicated ERG O'PROTACs (100 nM) and

harvested for western blot analysis $48 \mathrm{~h}$ post-transfection. ERK2 was used as a

loading control. (C) VCaP cells were transfected with a control or six indicated ERG

O'PROTACs (100 nM) and cells were harvested for western blot analysis $48 \mathrm{~h}$ post-

transfection. Both endogenous full-length (FL) wild-type and TMPRSS2-ERG (T2-

ERG) truncated ERG were detected. 


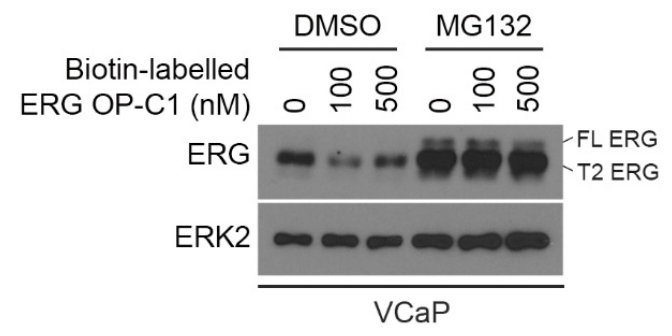

Figure 3. ERG O'PROTAC promotes ERG degradation via the protostome

pathway. VCaP cells were transfected with increasing concentrations of ERG OP-C1

for $36 \mathrm{~h}$, followed by treatment of the proteinase inhibitor MG132 for $12 \mathrm{~h}$ and western blot analysis.

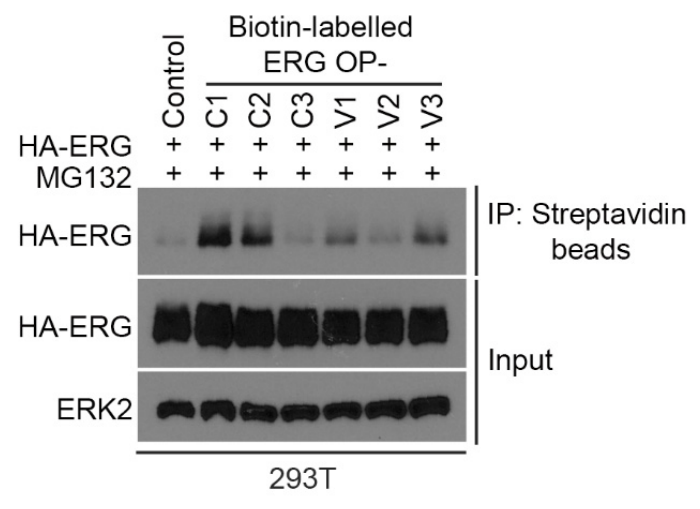

Figure 4. ERG O'PROTACs bind to ERG. 293T cells were transfected with HA-

ERG plasmid in combination with control (non-biotin labelled) or six indicated biotin-labelled ERG O'PROTACs (100 nM) and harvested for anti-biotin (streptavidin) pull-down assay $48 \mathrm{~h}$ post-transfection. 
A

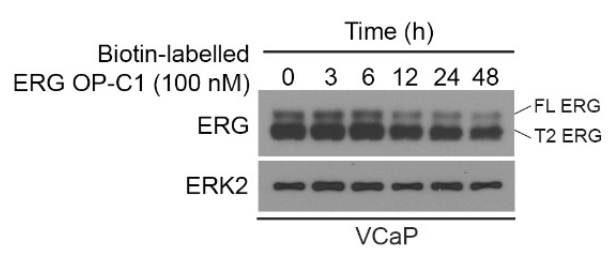

C

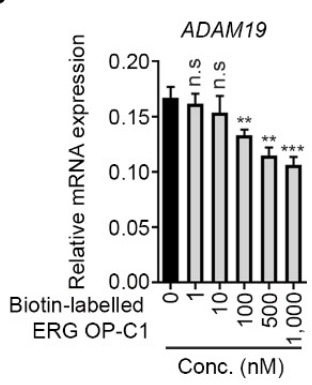

B

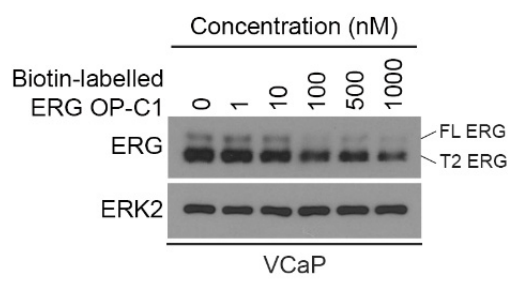

Figure 5. ERG O'PROTAC inhibits ERG transcriptional activity. (A) VCaP cells were transfected with $100 \mathrm{nM}$ of biotin-labelled ERG OP-C1. Cells were harvested at the different time points followed by western blot analysis. (B and C) VCaP cells were transfected with different concentrations of biotin-labelled ERG OP-C1 and harvested $45 \mathrm{~h}$ post-transfection for western blot analysis (B) and RT-qPCR analysis of mRNA expression of the indicated ERG-targeted genes (ADAM19, MMP3, MMP9, PLAT and $P L A U) . P$ values were calculated using the unpaired two-tailed Student's $t$ test; ${ }^{*} P<0.05 ; * * P<0.01 ; * * * P<0.001$, n.s., not significant.

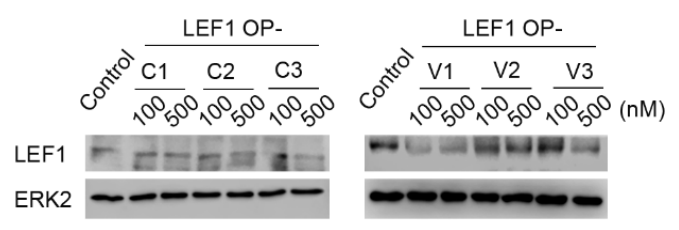

Figure 6. LEF-1 O'PROTACs degrade LEF1 protein in cultured cells. PC-3 cells were transfected with a control $(500 \mathrm{nM})$ or six indicated LEF1 O'PROTAC at different concentrations (100 and $500 \mathrm{nM}$ ) and cells were harvested for western blot 
analysis $48 \mathrm{~h}$ post-transfection. ERK2 was used as a loading control.

A

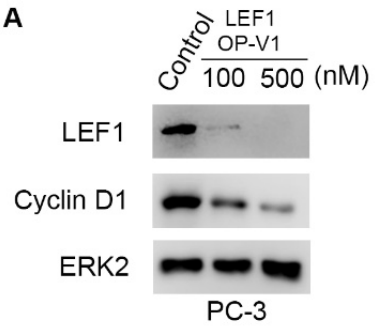

D

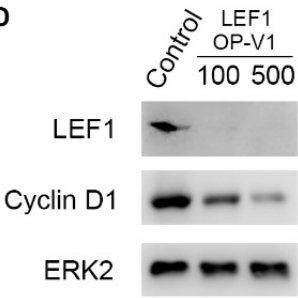

DU145
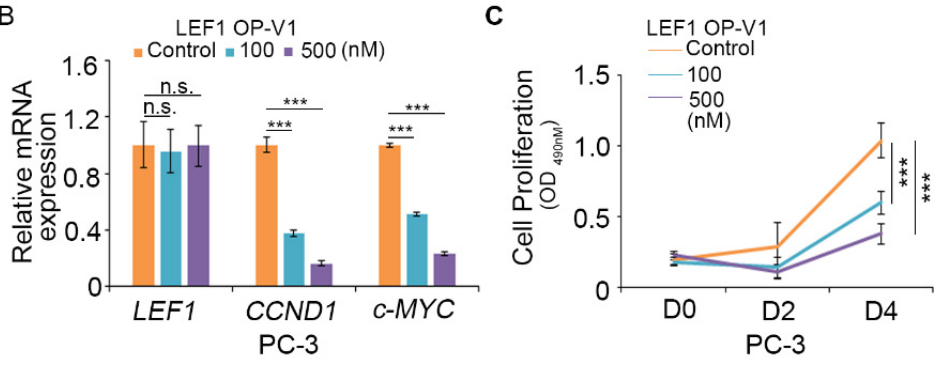

E

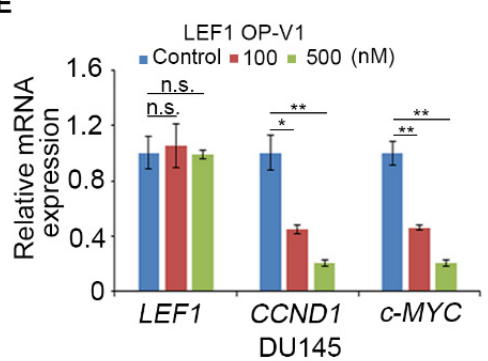

$\mathbf{F}$

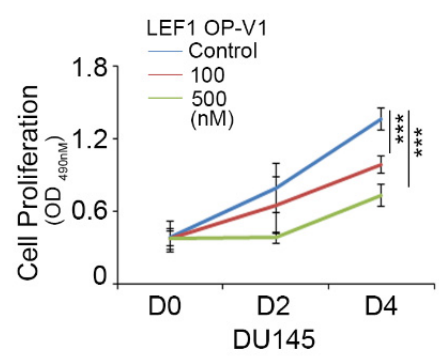

Figure 7. LEF1 O'PROTAC inhibits LEF1 target gene expression and prostate

cancer cell proliferation. (A-C) PC-3 cells were transfected with a control (500 nM)

or different concentrations of LEF1 OP-V1. At $48 \mathrm{~h}$ post-transfection cells were harvested for western blot analysis (A), RT-qPCR analysis of mRNA expression of LEF1 targeted genes (CCND1 and $c-M Y C)(\mathrm{B})$, and MTS assay at different days after treatment (C). (D-F) DU145 prostate cancer cells were transfected with a control (500 $\mathrm{nM}$ ) or different concentrations of LEF1 OP-V1. Transfected cells were subjected to western blot (D), RT-qPCR (E) and MTS assay (F). $P$ values were calculated using the unpaired two-tailed Student's $t$-test; $* P<0.05 ; * * P<0.01$; *** $P<0.001$, n.s., not significant. 


\section{References}

1. Lambert, M.; Jambon, S.; Depauw, S.; David-Cordonnier, M. H., Targeting Transcription Factors for Cancer Treatment. Molecules 2018, 23 (6).

2. Zhao, L.; Zhou, S.; Gustafsson, J.-Å., Nuclear Receptors: Recent Drug Discovery for Cancer Therapies. Endocrine Reviews 2019, 40 (5), 1207-1249.

3. Hagenbuchner, J.; Ausserlechner, M. J., Targeting transcription factors by small compounds-Current strategies and future implications. Biochemical Pharmacology 2016, 107, 1-13.

4. Burslem, G. M.; Song, J.; Chen, X.; Hines, J.; Crews, C. M., Enhancing Antiproliferative Activity and Selectivity of a FLT-3 Inhibitor by Proteolysis Targeting Chimera Conversion. Journal of the American Chemical Society 2018, 140 (48), 1642816432.

5. Cromm, P. M.; Samarasinghe, K. T. G.; Hines, J.; Crews, C. M., Addressing Kinase-Independent Functions of Fak via PROTAC-Mediated Degradation. Journal of the American Chemical Society 2018, 140 (49), 17019-17026.

6. Li, Z.; Lin, Y.; Song, H.; Qin, X.; Yu, Z.; Zhang, Z.; Dong, G.; Li, X.; Shi, X.; Du, L.; Zhao, W.; Li, M., First small-molecule PROTACs for G protein-coupled receptors: Inducing alpha1A-adrenergic receptor degradation. Acta Pharmaceutica Sinica $B \mathbf{2 0 2 0}$.

7. Khan, S.; Zhang, X.; Lv, D.; Zhang, Q.; He, Y.; Zhang, P.; Liu, X.; Thummuri, D.; Yuan, Y.; Wiegand, J. S.; Pei, J.; Zhang, W.; Sharma, A.; McCurdy, C. R.; Kuruvilla, V. M.; Baran, N.; Ferrando, A. A.; Kim, Y.-M.; Rogojina, A.; Houghton, P. J.; Huang, G.; Hromas, R.; Konopleva, M.; Zheng, G.; Zhou, D., A selective BCL-X(L) PROTAC degrader achieves safe and potent antitumor activity. Nat Med 2019, 25 (12), 19381947.

8. Neklesa, T.; Snyder, L. B.; Willard, R. R.; Vitale, N.; Pizzano, J.; Gordon, D. A.; Bookbinder, M.; Macaluso, J.; Dong, H.; Ferraro, C.; Wang, G.; Wang, J.; Crews, C. M.; Houston, J.; Crew, A. P.; Taylor, I., ARV-110: An oral androgen receptor PROTAC degrader for prostate cancer. Journal of Clinical Oncology 2019, 37 (7_suppl), 259-259.

9. Gao, H.; Sun, X.; Rao, Y., PROTAC Technology: Opportunities and Challenges. ACS Med Chem Lett 2020, 11 (3), 237-240.

10. Pettersson, M.; Crews, C. M., PROteolysis TArgeting Chimeras (PROTACs) - Past, present and future. Drug Discov Today Technol 2019, 31, 15-27.

11. Bai, L.; Zhou, H.; Xu, R.; Zhao, Y.; Chinnaswamy, K.; McEachern, D.; Chen, J.; Yang, C. Y.; Liu, Z.; Wang, M.; Liu, L.; Jiang, H.; Wen, B.; Kumar, P.; Meagher, J. L.; Sun, D.; Stuckey, J. A.; Wang, S., A Potent and Selective Small-Molecule Degrader of STAT3 Achieves Complete Tumor Regression In Vivo. Cancer Cell 2019, 36 (5), 498-511 e17.

12. Samarasinghe, K. T. G.; Jaime-Figueroa, S.; Dai, K.; Hu, Z.; Crews, C. M., Targeted Degradation of Transcription Factors by TRAFTACs. 2020.

13. Adamo, P.; Ladomery, M. R., The oncogene ERG: a key factor in prostate cancer. Oncogene 2016, 35 (4), 403-14. 
14. The Molecular Taxonomy of Primary Prostate Cancer. Cell 2015, 163 (4), 1011-25.

15. Perner, S.; Mosquera, J. M.; Demichelis, F.; Hofer, M. D.; Paris, P. L.; Simko, J.; Collins, C.; Bismar, T. A.; Chinnaiyan, A. M.; De Marzo, A. M.; Rubin, M. A., TMPRSS2-ERG fusion prostate cancer: an early molecular event associated with invasion. The American journal of surgical pathology 2007, 31 (6), 882-8.

16. Novak, A.; Dedhar, S., Signaling through beta-catenin and Lef/Tcf. Cellular and molecular life sciences : CMLS 1999, 56 (5-6), 523-37.

17. Kobayashi, W.; Ozawa, M., The transcription factor LEF-1 induces an epithelial-mesenchymal transition in MDCK cells independent of $\beta$-catenin. Biochemical and biophysical research communications 2013, 442 (1-2), 133-8.

18. Santiago, L.; Daniels, G.; Wang, D.; Deng, F.-M.; Lee, P., Wnt signaling pathway protein LEF1 in cancer, as a biomarker for prognosis and a target for treatment. American journal of cancer research 2017, 7 (6), 1389-1406.

19. Wei, G. H.; Badis, G.; Berger, M. F.; Kivioja, T.; Palin, K.; Enge, M.; Bonke, M.; Jolma, A.; Varjosalo, M.; Gehrke, A. R.; Yan, J.; Talukder, S.; Turunen, M.; Taipale, M.; Stunnenberg, H. G.; Ukkonen, E.; Hughes, T. R.; Bulyk, M. L.; Taipale, J., Genome-wide analysis of ETS-family DNA-binding in vitro and in vivo. EMBOJ 2010, 29 (13), 2147-60.

20. Cheng, J.; Li, Y.; Wang, X.; Dong, G.; Sheng, C., Discovery of Novel PDE $\delta$ Degraders for the Treatment of KRAS Mutant Colorectal Cancer. Journal of Medicinal Chemistry 2020, 63 (14), 7892-7905.

21. Crew, A. P.; Raina, K.; Dong, H.; Qian, Y.; Wang, J.; Vigil, D.; Serebrenik, Y. V.; Hamman, B. D.; Morgan, A.; Ferraro, C.; Siu, K.; Neklesa, T. K.; Winkler, J. D.; Coleman, K. G.; Crews, C. M., Identification and Characterization of Von HippelLindau-Recruiting Proteolysis Targeting Chimeras (PROTACs) of TANK-Binding Kinase 1. Journal of Medicinal Chemistry 2018, 61 (2), 583-598.

22. Tomlins, S. A.; Rhodes, D. R.; Perner, S.; Dhanasekaran, S. M.; Mehra, R.; Sun, X. W.; Varambally, S.; Cao, X.; Tchinda, J.; Kuefer, R.; Lee, C.; Montie, J. E.; Shah, R. B.; Pienta, K. J.; Rubin, M. A.; Chinnaiyan, A. M., Recurrent fusion of TMPRSS2 and ETS transcription factor genes in prostate cancer. Science 2005, 310 (5748), 644-8.

23. Liang, J.; Li, X.; Li, Y.; Wei, J.; Daniels, G.; Zhong, X.; Wang, J.; Sfanos, K.; Melamed, J.; Zhao, J.; Lee, P., LEF1 targeting EMT in prostate cancer invasion is mediated by miR-181a. American journal of cancer research 2015, 5 (3), 1124-32.

24. Edmondson, S. D.; Yang, B.; Fallan, C., Proteolysis targeting chimeras (PROTACs) in 'beyond rule-of-five' chemical space: Recent progress and future challenges. Bioorganic \& Medicinal Chemistry Letters 2019, 29 (13), 1555-1564.

25. Pan, X.; Rijnbeek, P.; Yan, J.; Shen, H. B., Prediction of RNA-protein sequence and structure binding preferences using deep convolutional and recurrent neural networks. BMC Genomics 2018, 19 (1), 511.

26. Wang, X.; Vukovic, L.; Koh, H. R.; Schulten, K.; Myong, S., Dynamic profiling of double-stranded RNA binding proteins. Nucleic Acids Res 2015, 43 (15), 7566-76. 
27. Li, X.; Kazan, H.; Lipshitz, H. D.; Morris, Q. D., Finding the target sites of RNA-binding proteins. Wiley Interdiscip Rev RNA 2014, 5 (1), 111-30.

28. Pal, A.; Levy, Y., Structure, stability and specificity of the binding of ssDNA and ssRNA with proteins. PLoS Comput Biol 2019, 15 (4), e1006768.

29. Sridharan, K.; Gogtay, N. J., Therapeutic nucleic acids: current clinical status. Br J Clin Pharmacol 2016, 82 (3), 659-672.

30. Lai, A. C.; Crews, C. M., Induced protein degradation: an emerging drug discovery paradigm. Nat Rev Drug Discov 2017, 16 (2), 101-114.

31. Roberts, T. C.; Langer, R.; Wood, M. J. A., Advances in oligonucleotide drug delivery. Nat Rev Drug Discov 2020, 19 (10), 673-694.

32. Chung, J. Y.; Thone, M. N.; Kwon, Y. J., COVID-19 vaccines: The status and perspectives in delivery points of view. Adv Drug Deliv Rev 2020, 170, 1-25. 